\title{
Complications following symptom-limited thoracocentesis using suction
}

Thoracocentesis is a common pleural intervention and these interventions are not without possible complication. Pneumothorax and re-expansion pulmonary oedema (REPO) are among the most feared complications. Several strategies have been proposed to avoid complications and include avoidance of suction for drainage, limitation of volume removed, and drainage guided by pleural manometry.

Standard guidelines recommend removing less than $1.5 \mathrm{~L}$ and to not use suction drainage. There is a paucity of supporting evidence for these recommendations, as most published literature on thoracocentesis includes manual aspiration of pleural fluid. Additionally, it is recommended to drain less than $300 \mathrm{~mL}$ of fluid if there is ipsilateral mediastinal shift. None of these recommendations consider the volume of fluid drained in relation to the size of the chest cavity.

The purpose of this study was to examine the incidence of thoracocentesis-related complications, including pneumothorax and $\mathrm{REPO}$, in patients undergoing symptom-limited suction drainage. A secondary aim was to identify risk factors associated with REPO.

This was a retrospective cohort study of all thoracocenteses in adult patients performed by the Department of Pulmonary Medicine at The University of Texas MD Anderson Cancer Center (Houston, TX, USA) between 1 January 12004 and 31 August 2018. A thoracic safety needle was introduced via sterile technique under local anaesthetic and an $8 \mathrm{Fr}$ catheter was introduced into the pleural space. This was attached to a $1 \mathrm{~L}$ vacuum bottle (before May 2009) and to a $2 \mathrm{~L}$ plastic cannister attached to wall suctioning at maximal setting (after May 2009). The volume of fluid aspirated was normalised by estimated lung capacity of the affected hemithorax (V/LC).

Pneumothorax was documented if it was visualised on subsequent chest radiograph (pneumothorax ex vacuo was also included as pneumothorax). REPO was diagnosed under the following conditions: (i) acute clinical decompensation; (ii) diagnostic chest imaging findings compatible with REPO; and (iii) within 48 hours of thoracocentesis.

The final cohort included 7206 patients, which constituted 10344 thoracocenteses. REPO was confirmed in only eight patients $(0.08 \%)$. The incidence of REPO increased to $0.30 \%$ (95\% CI 0.05 - 1.23) when draining $\geq 1.5 \mathrm{~L}$ and to $0.61 \%$ (95\% CI 0.15 - 2.35) when draining $\geq 2$ L. In patients with ECOG PS 3 or 4 , the incidence of REPO increased to $0.54 \%$ ( $95 \%$ CI 0.13 - 2.06) when draining $\geq 1.5 \mathrm{~L}$ and to $1.29 \%$ (95\% CI 0.31 - 5.53) when draining $\geq 2$ L. Most of the REPO cases (seven out of eight) did not have mediastinal shift. Patients who had an ipsilateral shift in the mediastinum had less volume drained when compared with patients with central and contralateral mediastinal shift (median 850 v. $1000 \mathrm{~mL} ; p<0.01$ ). In patients with pre-procedure ipsilateral deviation of the mediastinum, there was no increase in the incidence of post-procedure pneumothorax (40 v. 372; $p=0.18$ ) or pneumothorax requiring intervention ( 2 v. $27 ; p=1.00)$.

This study demonstrated that symptom-limited suction drainage of pleural fluid was a safe intervention and there was a low risk of complications even in cases where large volumes were drained. The incidence of REPO was $0.08 \%$; in patients with drainage $\geq 2 \mathrm{~L}$, the highest estimated probability was $2.35 \%$. Patients with poor ECOG PS had a significantly increased risk of REPO with removal of $\geq 1.5 \mathrm{~L}$ or $\mathrm{V} / \mathrm{LC}>0.6$.

There were $0.61 \%$ ( $n=412 / 11344$ thoracocenteses) iatrogenic pneumothoraces and they were associated with removal of more than $1.5 \mathrm{~L}$ of fluid $(p<0.01)$, unilateral procedures $(p=0.01)$ and repeated needle pass through skin ( $p=0.03$ ). Only 29 out of 412 cases needed further intervention and, as a post-procedure chest radiograph was not requested in some cases, asymptomatic pneumothoraces may have been missed.

Restriction of fluid drainage based on volume is not warranted. However, caution should be exercised in patients with a poor ECOG PS if large volumes are drained.

\section{Andre Nortje}

Division of Pulmonology, Department of Medicine, Stellenbosch University, Cape Town, South Africa

andre_nortje@yahoo.com

\footnotetext{
1. Sagar AES, Landaeta MF, Adrianza AM, et al. Complications following symptomlimited thoracentesis using suction. Eur Respir J 2020;56:1902356 https://doi. org/10.1183/13993003.02356-2019
} 